\title{
Genetic Basis Of Arrhythmogenic Cardiomyopathy
}

\author{
Jennifer Karmouch $^{\mathrm{a}}$, Alexandros Protonotarios ${ }^{\mathrm{b}}$, and Petros Syrris ${ }^{\mathrm{b}}$
}

${ }^{a}$ Center for Cardiovascular Genetics, Institute of Molecular Medicine, The University of Texas Health Sciences Center, Houston, USA

${ }^{\mathrm{b}}$ Centre for Heart Muscle Disease, Institute of Cardiovascular Science, University College London, London, UK

Correspondence to Dr Jennifer Karmouch, PhD, Center for Cardiovascular Genetics, 6770 Bertner Street, Suite C900A, Houston, TX 77030, USA

Tel: +1 7135002307

Email: Jennifer.Karmouch@uth.tmc.edu

\section{Purpose of review}

To date 16 genes have been associated with arrhythmogenic cardiomyopathy (ACM). Mutations in these genes can lead to a broad spectrum of phenotypic expression ranging from disease affecting predominantly the right or left ventricle, to bi-ventricular subtypes. Understanding the genetic causes of arrhythmogenic cardiomyopathy is important in diagnosis and management of the disorder. This review summarizes recent advances in molecular genetics and discusses the application of next generation sequencing technology in genetic testing in ACM.

\section{Recent findings}


Use of next generation sequencing methods has resulted in the identification of novel causative variants and genes for arrhythmogenic cardiomyopathy. The involvement of FLNC in ACM demonstrates the genetic overlap between arrhythmogenic cardiomyopathy and other types of cardiomyopathy. Putative pathogenic variants have been detected in $\mathrm{CDH} 2$, the gene encoding cadherin 2, a protein involved in cell adhesion. Large genomic rearrangements in desmosome genes have been systematically investigated in a cohort of ACM patients.

\section{Summary}

Recent studies have identified novel causes of arrhythmogenic cardiomyopathy providing new insights into the genetic spectrum of the disease and highlighting an overlapping phenotype between arrhythmogenic cardiomyopathy and dilated cardiomyopathy. Next generation sequencing is a useful tool for research and genetic diagnostic screening but interpretation of identified sequence variants requires caution and should be performed in specialized centers.

\section{Keywords}

Arrhythmogenic cardiomyopathy, arrhythmogenic right ventricular cardiomyopathy, genetics, mutations, next generation sequencing

\section{INTRODUCTION}

Arrhythmogenic cardiomyopathy (ACM) is a rare, primary heart muscle disorder, presenting with increased risk for ventricular arrhythmias and sudden cardiac death. Histologically, ACM is characterized by loss of myocardial tissue and its replacement by fibrofatty tissue. [1] ACM affects approximately 1 in 2000 to 5000 individuals and is considered one of the major causes 
of arrhythmic sudden cardiac death in the young and athletes. [1] It is typically inherited as an autosomal dominant disorder but a small number of recessive cases are also known. [2*]

The disorder was originally termed "arrhythmogenic right ventricular dysplasia" (ARVD) suggesting the dysplastic nature of the disease. [3] ACM patients have a structurally normal heart at birth and develop the cardiomyopathy changes later on. It was later named "arrhythmogenic right ventricular cardiomyopathy" (ARVC) because it was believed that the disease predominantly affects the right ventricle. [1] This is only true for classic disease subtypes and the recognition of other forms, i.e. predominant left-sided or bi-ventricular cardiomyopathy, has led to the adoption of the broader term "arrhythmogenic cardiomyopathy".

The purpose of this review is to aid physicians to better understand the genetic basis of $\mathrm{ACM}$, review publications in the past year and highlight recent findings that increase our knowledge of the genetic causes related to development of this complex disease, and discuss the use of next generation sequencing in the genetic diagnosis of ACM.

\section{GENETIC BASIS}

\section{Known genes linked to ACM}

The key to the discovery of the genetic causes of ACM was its association with palmoplantar keratoderma and woolly hair in the description of Naxos Disease, named after the Greek island, where the first observed families resided. [4] The syndrome is inherited in an autosomal recessive manner with full penetrance by adolescence that facilitated genetic linkage analysis and identification of homozygosity for a two-nucleotide deletion (2157del2) in plakoglobin $(J U P)$ as the underlying genetic cause. [5] 
Soon after, mutations in other genes encoding main components of the desmosome were found to cause ACM, including desmoplakin (DSP), plakophilin-2 (PKP2), desmoglein2 (DSG2) and desmocollin-2 (DSC2). [6-9] Desmosomes are complex structures consisting of proteins and are responsible for cell adhesion and signalling. [10] Thus, abnormalities in desmosome structure were justifiably considered to have a key role in ACM pathogenesis and the classic form of the disease presenting with right ventricular cardiomyopathy was considered a "disease of the desmosome". [11] Approximately 30-50\% of ACM patients carry a pathogenic sequence variant in one of these key desmosome genes (Table 1). In the majority of cases inheritance is in an autosomal dominant manner with incomplete penetrance and variable expressivity. [22]

Mutations in other, non-desmosomal genes have been have been found in families with ACM (Table 1). These include: transforming growth factor $\beta 3$ (TGF- $\beta 3$ ); transmembrane protein 43 (TMEM43); alpha T-catenin (CTNNA3); titin (TTN); lamin A/C (LMNA); phospholamban $(P L N)$ and desmin $(D E S)$. [12, 14,15, 17-20] Such findings in genes previously linked to other forms of cardiomyopathies, represent an overlap between ACM and dilated cardiomyopathy (DCM) both at the clinical and genetic level. Finally, it is also worth noting that mutations in the cardiac ryanodine receptor $2(R Y R 2)$ gene have also been reported in ACM [23] but it is now recognised that the observed phenotype is catecholamine-induced ventricular tachycardia rather than ACM and this gene is no longer considered as ACM causing.

Genetic studies can identify a causative mutation in up to $60 \%$ of ACM cases. In the largest study of ACM patients and family members to date, $37 \%$ of cases were negative for mutations in desmosome genes, TMEM43 or PLN. [24] It is postulated that gene-negative ACM cases could possibly harbour mutations in unidentified genes or non-coding parts of the genome or have large deletions/duplications. It is also possible that disease in those individuals could 
be the result of a combination of unknown low-penetrance genetic variants and environmental factors which would point to an oligogenic form of ACM, as reported in human HCM. (25)

\section{New genes linked to ACM}

In the last year three new genes have been implicated in the pathogenesis of ACM. Ortiz-Genga et al. [16**] used next generation sequencing to screen 2,877 patients with inherited cardiovascular disease. They identified 23 truncating mutations in the filamin $\mathrm{C}(F L N C)$ gene in probands with dilated and left-dominant arrhythmogenic cardiomyopathy. Filamin $\mathrm{C}$ is expressed in striated muscle where it interacts with the actin cytoskeleton and other sarcomere proteins providing stability and signalling functions. [26] FLNC mutations have been previously associated with dilated cardiomyopathy [27] but the study by Ortiz-Genga et al. [16**] clearly demonstrated how ACM and arrhythmogenic forms of DCM overlap in the clinical and genetic level.

Using whole exome sequencing two groups independently identified cadherin 2 $(\mathrm{CDH} 2)$ as a novel ACM gene. [13*,28] The number of ACM patients included in those studies was relatively small (single patient in one and 73 genotype-negative probands in the other) and only two $\mathrm{CDH} 2$ putatively disease-causing variants were detected in total. However, cadherin 2 is a biologically plausible candidate gene for ACM as it plays an important role in cell adhesion. [29] Along with alpha T-catenin, a gene already implicated in ACM, cadherin 2 is expressed in the area composita, a specialized region of the intercalated discs which connect cardiomyocytes to one another.

Interestingly, a recent study has proposed that the voltage-gated sodium channel subunit alpha Nav1.5 (SCN5A) gene is associated with ACM. [21*] The authors detected a putatively pathogenic SCN5A sequence variant in 5/281 patients (1.8\%) fulfilling diagnostic criteria for arrhythmogenic right ventricular cardiomyopathy. Those variants were associated with prolonged QRS duration and their carriers had major structural abnormalities on cardiac 
imaging. [21*] Furthermore, based on functional studies performed on one variant (Arg1898His), the authors suggest that Nav1.5 is in a functional complex with adhesion molecules such as $\mathrm{N}$-cadherin. This would potentially indicate that Nav1.5 dysfunction causes ACM via non-canonical mechanisms. If validated in larger studies, these findings could have important implications on our understanding of the pathogenesis of ACM.

\section{Large genomic rearrangements linked to ACM}

Until recently, copy number variations (CNVs, i.e. large genomic rearrangements such as gross deletions, insertions and duplications) in the $P K P 2$ gene have been reported in case reports as the cause of arrhythmogenic cardiomyopathy. [30,31] Pilichou et al. [32**] adopted a systematic approach to determine the prevalence of CNVs in the five key desmosome genes in ACM patients negative for point mutations in these genes. Investigation of 160 index cases revealed 11 CNVs (6.9\%) in $P K P 2$, but also in DSC2 and DSG2 genes. It should be noted however that only $32 \%$ of family members carrying the CNV fulfilled the diagnostic criteria. Regardless, these results support CNV screening as potential causes of ACM.

\section{NEXT GENERATION SEQUENCING SCREENING AND ACM}

Recent advances in DNA sequencing techniques have revolutionized cardiovascular genetics facilitating rapid, accurate and cost-effective screening of human DNA for variation on the whole genome scale. Three different approaches are available: targeted sequencing of specific gene panels; whole exome sequencing (WES) and whole genome sequencing. In practice, for inherited cardiovascular diseases and in particular for ACM, only the first two methods are currently used for diagnostic purposes and research in order to identify known as well as novel causative variants and genes.

Given the emerging, common genetic causes and overlapping phenotypes amongst different types of cardiomyopathy, including ACM, many groups have designed broad targeted 
gene panels that include not just ACM-associated genes but also known causative genes for other cardiomyopathies. For example, a recent study which identified $F L N C$ as disease-causing in ACM, used a panel of 213 genes related to inherited cardiovascular diseases and sudden death [16**], whilst Hertz et al. [33] screened 100 genes previously associated with inherited cardiomyopathies and channelopathies. In another study of a small cohort of 14 patients, analysis focused on 96 known genes and found potentially pathogenic variants in $64 \%$ of cases. $[34 *]$

However, the targeted gene approach can only detect variants in known genes and therefore patients with no identified mutation must be screened again by WES which theoretically covers the entire human exome. WES has been successful in finding new ACM genes $\left[13^{*}\right]$ and is mainly used for research rather than diagnostic screening. This is mainly due to the serious difficulties in interpretation of WES genetic data in general. Classification of genetic variants according to their predicted pathogenicity remains challenging as current bioinformatics software used for this purpose lack specificity whilst many rare ACM variants are also present in the general population [35, 36], a fact that raises doubts about whether they can be genuinely disease-causing. Such variants are currently classified as "variants of unknown significance" (VUS). Consequently, interpretation of rare and/or novel genetic variants in ACM cases should be performed with caution as their pathogenicity cannot be fully ascertained.

\section{CHALLENGES IN GENETIC TESTING}

The role of genetic testing in ACM is fundamental in identifying a pathogenic mutation in an index case and subsequently determining the potential risk in their family members. So much so that the presence of a pathogenic mutation has been classified as a major criterion in the 2010 modification of the Task Force criteria for the diagnosis of ACM. [37] 
However, utilization of genetic results in daily clinical practice is hindered by a number of factors. The main ones include: ACM presents with incomplete, age-related penetrance and variable phenotypic expression; a proportion of cases carry more than one variant in the same or different genes; as described earlier in this review, variants detected in ACM cases have also been found in apparently healthy individuals; and, finally, our incomplete understanding of the functional significance of sequence variants obtained by genetic screening. As other cardiac disorders can mimic certain phenotypic features of ACM, in some index cases gene testing should not be used as the only diagnostic tool-

These limitations highlight the necessity to perform genetic testing and review the resulting genetic data in expert cardiovascular centers.

\section{PATHOGENESIS}

Within the heart, desmosome proteins are known to be expressed primarily in cardiac myocytes. The deletion of the Dsp gene in cardiac myocytes leads to cardiac dysfunction, cardiac arrhythmias, premature death, and fibro-adipogenesis (38). Research over several years in mouse models of ACM has provided insights into the cellular origin and mechanisms of ACM. Recent studies have shown that cells other than cardiac myocytes contribute to the pathogenesis of ACM (39-41). Specifically, fibroadipocyte progenitor cells, marked by the expression of platelet derived growth factor alpha and other markers, were shown to express desmosome proteins and upon deletion of $D s p$ differentiate to fibroadipocytes in ACM (40). Furthermore, deletion of desmoplakin in cells of the cardiac conduction system and skin keratinocytes, marked by the expression of chondroitin sulfate proteoglycan 4 leads to cardiac arrhythmias, palmoplantar keratosis, and alopecia, as observed in Naxos disease (41). Mechanistic studies using these models identified a critical role of the canonical WNT and Hippo pathways in fibroadipogenesis in $\operatorname{ACM}(38,40,42-44)$. Taken together these findings 
provide plausible mechanisms and expand the current view of the pathogenesis of ACM, supporting a multicellular origin of its phenotypes.

\section{GENOTYPE-PHENOTYPE CORRELATIONS}

Studies of patients with ACM have shown high genetic heterogeneity and limited genotypephenotype correlations. [45] Disease expression can vary significantly amongst ACM patients, even in those harbouring mutations in the same gene or carrying the same mutation. [46] $\mathrm{A}$ large study of over 1,000 ACM patients and family members found no statistically significant difference in clinical characteristics and outcomes between probands with and without mutations, as well as those with familial and non-familial disease. [24] However, patients with multiple mutations (homozygotes, compound heterozygotes, or digenic mutation carriers) generally exhibit a more severe phenotype $[47,48]$ and the presence of multiple desmosome gene variants is a significant risk factor for major arrhythmic events and sudden cardiac death. [48] In addition, patients with multiple variants have been demonstrated to develop significantly earlier onset of symptoms and occurrence of sustained ventricular arrhythmias. [49]

A recent meta-analysis of eleven genotype-phenotype relationship studies revealed that mutations in genes encoding desmosome proteins were associated with an early age of onset of the disease, presence of $\mathrm{T}$ wave inversion in the precordial leads, and a family history of ACM. [50] On the other hand, presence and severity of cardiac structural abnormalities, epsilon wave, and ventricular tachycardia were similar in those with and without mutations in genes encoding desmosome proteins. Finally, Castelletti et al. [51] compared groups of ACM patients carrying missense and non-missense mutations in desmoplakin. Non-missense DSP mutations were particularly associated with left-dominant forms of the disease as left ventricular dysfunction and left ventricular structural abnormalities were significantly more common in patients with non-missense mutations compared to those with missense variants. [51] 


\section{CONCLUSION}

$\mathrm{ACM}$ is an inherited cardiomyopathy characterized by genetic heterogeneity, incomplete penetrance and variable phenotypic expression. Mutations in known ACM genes only account approximately for $60 \%$ of patients suggesting that other, yet unidentified, genetic defects could be the cause of the condition in the remaining cases.

Recent studies expanded the genetic spectrum of ACM by implicating a further 3 genes, $F L N C, C D H 2$ and $S C N 5 A$, in the pathogenesis of the disorder. The discovery of ACM-causing mutations in $F L N C$, a gene causative in other cardiomyopathies too, established beyond doubt that ACM shares genetic background and clinical presentation with arrhythmogenic forms of

DCM. The studies on $\mathrm{CDH} 2$ are in agreement with current knowledge that defects in cell adhesion genes can lead to ACM. The role of SCN5A in ACM is still not fully understood and studies in larger patient cohorts are required in order to elucidate how mutations in this gene can cause ACM.

Genetic testing plays an important role in detecting potentially pathogenic mutations in probands and identifying relatives at risk of developing the disorder. In that respect next generation sequencing methods are a useful tool in investigating the genetic basis of ACM but interpretation of genetic results requires specialized knowledge and expertise.

\section{KEY POINTS}

* ACM is a rare primary myocardial disease and an important cause of sudden cardiac death in the young. 
* Sixteen genes have been implicated in ACM but mutations in those only account for approximately $60 \%$ of cases.

* Recent discoveries of new causative genes such as FLNC have broadened the genetic spectrum of the disease and highlighted the genetic and phenotypic overlap between ACM and other types of cardiomyopathy, particularly DCM.

* There is evidence of genotype-phenotype correlations with limited clinical utility at present.

* Interpretation of genetic data obtained by next generation sequencing remains challenging and should be performed in expert centers.

\section{Acknowledgements}

This work was funded by Fondation Leducq Transatlantic Networks of Excellence Program: GRANT no 14 CVD 03 and supported by the National Institute for Health Research University College London Hospitals Biomedical Research Centre.

\section{Conflicts of interest}

There are no conflicts of interest

\section{REFERENCES AND RECOMMENDED READING}

1. Basso C, Corrado D, Marcus FI, et al. Arrhythmogenic right ventricular cardiomyopathy. Lancet 2009; 373:1289-1300. 
2. Hoorntje ET, te Rijdt WP, James CA, et al. Arrhythmogenic cardiomyopathy: pathology, genetics, and concepts in pathogenesis. Cardiovasc Res 2017; 113:15211531.

* This manuscript provides a thorough review of the pathogenic mechanisms in ACM.

3. Marcus FI, Fontaine GH, Guiraudon G, et al. Right ventricular dysplasia: a report of 24 adult cases. Circulation 1982; 65:384-398.

4. Protonotarios N, Tsatsopoulou A, Patsourakos $\mathrm{P}$, et al. Cardiac abnormalities in familial palmoplantar keratosis. Br Heart J 1986; 56:321-326.

5. McKoy G, Protonotarios N, Crosby A, et al. Identification of a deletion in plakoglobin in arrhythmogenic right ventricular cardiomyopathy with palmoplantar keratoderma and woolly hair (Naxos disease). Lancet 2000; 355:2119-2124.

6. Rampazzo A, Nava A, Malacrida S, et al. Mutation in human desmoplakin domain binding to plakoglobin causes a dominant form of arrhythmogenic right ventricular cardiomyopathy. Am J Hum Genet 2002; 71:1200-1206.

7. Gerull B, Heuser A, Wichter T, et al. Mutations in the desmosomal protein plakophilin2 are common in arrhythmogenic right ventricular cardiomyopathy. Nat Genet 2004; $36: 1162-1164$.

8. Pilichou K, Nava A, Basso C, et al. Mutations in desmoglein-2 gene are associated with arrhythmogenic right ventricular cardiomyopathy. Circulation 2006; 113:1171-1179.

9. Syrris P, Ward D, Evans A, et al. Arrhythmogenic right ventricular dysplasia/cardiomyopathy associated with mutations in the desmosomal gene desmocollin-2. Am J Hum Genet 2006; 79:978-984.

10. Huber O. Structure and function of desmosomal proteins and their role in development and disease. Cell Mol Life Sci 2003; 60:1872-1890. 
11. Sen-Chowdhry S, Syrris P, McKenna WJ. Genetics of right ventricular cardiomyopathy. J Cardiovasc Electrophysiol 2005; 16:927-935.

12. van Hengel J, Calore M, Bauce B, et al. Mutations in the area composita protein alphaTcatenin are associated with arrhythmogenic right ventricular cardiomyopathy. Eur Heart J 2013; 34:201-210.

13. Mayosi BM, Fish M, Shaboodien G, et al. Identification of cadherin 2 (CDH2) mutations in arrhythmogenic right ventricular cardiomyopathy. Circ Cardiovasc Genet 2017; 10:e001605.

* This study identified mutations in $\mathrm{CDH} 2$ further supporting the view that defects in cell adhesion genes cause ACM.

14. Merner ND, Hodgkinson KA, Haywood AF, et al. Arrhythmogenic right ventricular cardiomyopathy type 5 is a fully penetrant, lethal arrhythmic disorder caused by a missense mutation in the TMEM43 gene. Am J Hum Genet 2008; 82:809-821.

15. Taylor M, Graw S, Sinagra G, et al. Genetic variation in titin in arrhythmogenic right ventricular cardiomyopathy-overlap syndromes. Circulation 2011; 124:876-885.

16. Ortiz-Genga MF, Cuenca S, Dal Ferro M, et al. Truncating FLNC mutations are associated with high-risk dilated and arrhythmogenic cardiomyopathies. J Am Coll Cardiol 2016; 68:2440-2451.

** This study identified FLNC as a disease-causing gene in ACM and highlighted the genetic and overlap between ACM and DCM.

17. Quarta G, Syrris P, Ashworth M, et al. Mutations in the Lamin A/C gene mimic arrhythmogenic right ventricular cardiomyopathy. Eur Heart J 2012; 33:1128-1136.

18. Beffagna G, Occhi G, Nava A, et al. Regulatory mutations in transforming growth factor-beta3 gene cause arrhythmogenic right ventricular cardiomyopathy type 1 . Cardiovasc Res 2005; 65:366-373. 
19. van der Zwaag PA, van Rijsingen IA, Asimaki A, et al. Phospholamban R14del mutation in patients diagnosed with dilated cardiomyopathy or arrhythmogenic right ventricular cardiomyopathy: evidence supporting the concept of arrhythmogenic cardiomyopathy. Eur J Heart Fail 2012; 14:1199-1207.

20. van Tintelen JP, Van Gelder IC, Asimaki A, et al. Severe cardiac phenotype with right ventricular predominance in a large cohort of patients with a single missense mutation in the DES gene. Heart Rhythm 2009; 6:1574-1583.

21. Te Riele AS, Agullo-Pascual E, James CA, et al. Multilevel analyses of SCN5A mutations in arrhythmogenic right ventricular dysplasia/cardiomyopathy suggest noncanonical mechanisms for disease pathogenesis. Cardiovasc Res 2017; 113:102-111.

* First study to implicate SCN5A in ACM.Tiso N, Stephan DA, Nava A, et al. Identification of mutations in the cardiac ryanodine receptor gene in families affected with arrhythmogenic right ventricular cardiomyopathy type 2 (ARVD2). Hum Mol Genet 2001; 10:189-194.

22. Corrado D, Basso C, Pilichou K, et al. Molecular biology and clinical management of arrhythmogenic right ventricular cardiomyopathy/dysplasia. Heart 2011; 97:530-539.

23. Tiso N, Stephan DA, Nava A, et al. Identification of mutations in the cardiac ryanodine receptor gene in families affected with arrhythmogenic right ventricular cardiomyopathy type 2 (ARVD2). Hum Mol Genet 2001; 10:189-194.

24. Groeneweg JA, Bhonsale A, James CA, et al. Clinical presentation, long-term followup, and outcomes of 1001 arrhythmogenic right ventricular dysplasia/cardiomyopathy patients and family members. Circ Cardiovasc Genet 2015; 8:437-446.

25. Li L, Bainbridge MN, Tan Y, Willerson JT, et al. A Potential Oligogenic Etiology of Hypertrophic Cardiomyopathy: A Classic Single-Gene Disorder. Circ Res. 2017; $7: 1084-1090$ 
26. Fürst DO, Goldfarb LG, Kley RA, et al. Filamin C-related myopathies: pathology and mechanisms. Acta Neuropathol 2013; 125:33-46.

27. Begay RL, Tharp CA, Martin A, et al. FLNC gene splice mutations cause dilated cardiomyopathy. JACC Basic Transl Sci 2016; 1:344-359.

28. Turkowski KL, Tester DJ, Bos JM, et al. Whole exome sequencing with genomic triangulation implicates $\mathrm{CDH} 2$-encoded $\mathrm{N}$-cadherin as a novel pathogenic substrate for arrhythmogenic cardiomyopathy. Congenit Heart Dis 2017; 12:226-235.

29. Sheikh F, Ross RS, Chen J. Cell-cell connection to cardiac disease. Trends Cardiovasc Med 2009; 19:182-190.

30. Roberts JD, Herkert JC, Rutberg J, et al. Detection of genomic deletions of PKP2 in arrhythmogenic right ventricular cardiomyopathy. Clin Genet 2013; 83:452-456.

31. Li Mura IE, Bauce B, Nava A, et al. Identification of a PKP2 gene deletion in a family with arrhythmogenic right ventricular cardiomyopathy. Eur J Hum Genet 2013; 21:1226-1231.

32. Pilichou K, Lazzarini E, Rigato I, et al. Large genomic rearrangements of desmosomal genes in italian arrhythmogenic cardiomyopathy patients. Circ Arrhythm Electrophysiol 2017; 10: e005324.

** First systematic study of desmosome gene CNVs in ACM.

33. Hertz CL, Christiansen SL, Ferrero-Miliani L, et al. Next-generation sequencing of 100 candidate genes in young victims of suspected sudden cardiac death with structural abnormalities of the heart. Int J Legal Med 2016; 130:91-102.

34. Medeiros-Domingo A, Saguner AM, Magyar I, et al. Arrhythmogenic right ventricular cardiomyopathy: implications of next-generation sequencing in appropriate diagnosis. Europace 2017; 19:1063-1069.

* This study uses WES for screening of ACM cases. 
35. Andreasen C, Nielsen JB, Refsgaard L, et al. New population-based exome data are questioning the pathogenicity of previously cardiomyopathy-associated genetic variants. Eur J Hum Genet 2013; 21:918-928.

36. Lahtinen AM, Lehtonen E, Marjamaa A, et al. Population-prevalent desmosomal mutations predisposing to arrhythmogenic right ventricular cardiomyopathy. Heart Rhythm 2011; 8:1214-1221.

37. Marcus FI, McKenna WJ, Sherrill D, et al. Diagnosis of arrhythmogenic right ventricular cardiomyopathy/dysplasia: proposed modification of the task force criteria. Circulation 2010; 121:1533-1541.

38. Garcia-Gras E, Lombardi R, Giocondo MJ, et al. Suppression of canonical Wnt/betacatenin signaling by nuclear plakoglobin recapitulates phenotype of arrhythmogenic right ventricular cardiomyopathy. J Clin Invest. 2006; 116:2012-2021.

39. Sommariva E, Brambilla S, Carbucicchio C, et al. Cardiac mesenchymal stromal cells are a source of adipocytes in arrhythmogenic cardiomyopathy. Eur Heart J. 2016; $37: 1835-1846$

40. Lombardi R, Chen SN, Ruggiero A, et al. Cardiac fibro-adipocyte progenitors express desmosome proteins and preferentially differentiate to adipocytes upon deletion of the desmoplakin gene. Circ Res. 2016; 119:41-54.

41. Karmouch J, Zhou QQ, Miyake CY, et al. Distinct Cellular Basis for Early Cardiac Arrhythmias, the Cardinal Manifestation of Arrhythmogenic Cardiomyopathy, and the Skin Phenotype of Cardiocutaneous Syndromes. 2017; 121:1346-1359.

42. Lombardi R, Dong J, Rodriguez G, et al. Genetic fate mapping identifies second heart field progenitor cells as a source of adipocytes in arrhythmogenic right ventricular cardiomyopathy. 2009; 104:1076-1084. 
43. Lombardi R, da Graca Cabreira-Hansen M, Bell A, et al. Nuclear plakoglobin is essential for differentiation of cardiac progenitor cells to adipocytes in arrhythmogenic right ventricular cardiomyopathy. 2011; 109:1342-53.

44. Chen SN, Gurha P, Lombardi R, et al. The hippo pathway is activated and is a causal mechanism for adipogenesis in arrhythmogenic cardiomyopathy. Circ Res. 2014; $114: 454-468$.

45. Sen-Chowdhry S, Syrris P, McKenna WJ. Role of genetic analysis in the management of patients with arrhythmogenic right ventricular dysplasia/cardiomyopathy. J Am Coll Cardiol 2007; 50:1813-1821.

46. Syrris P, Ward D, Asimaki A, et al. Desmoglein-2 mutations in arrhythmogenic right ventricular cardiomyopathy: a genotype-phenotype characterization of familial disease. Eur Heart J 2007; 28:581-588.

47. Xu T, Yang Z, Vatta M, et al. Compound and digenic heterozygosity contributes to arrhythmogenic right ventricular cardiomyopathy. J Am Coll Cardiol 2010; 55:587597.

48. Rigato I, Bauce B, Rampazzo A, et al. Compound and digenic heterozygosity predicts lifetime arrhythmic outcome and sudden cardiac death in desmosomal gene-related arrhythmogenic right ventricular cardiomyopathy. Circ Cardiovasc Genet 2013; 6:533542.

49. Bhonsale A, Groeneweg JA, James CA, et al. Impact of genotype on clinical course in arrhythmogenic right ventricular dysplasia/cardiomyopathy-associated mutation carriers. Eur Heart J 2015; 36:847-855.

50. Xu Z, Zhu W, Wang C, et al. Genotype-phenotype relationship in patients with arrhythmogenic right ventricular cardiomyopathy caused by desmosomal gene mutations: A systematic review and meta-analysis. Sci Rep 2017; 7:41387. 
51. Castelletti S, Vischer AS, Syrris P, et al. Desmoplakin missense and non-missense mutations in arrhythmogenic right ventricular cardiomyopathy: Genotype-phenotype correlation. Int J Cardiol 2017; 249:268-273. 
Table 1. Genes implicated in ACM

\begin{tabular}{|c|c|c|c|c|}
\hline Gene & Protein & $\begin{array}{c}\text { Chromosomal } \\
\text { location }\end{array}$ & $\begin{array}{c}\text { Estimated } \\
\text { prevalence } \\
(\%)\end{array}$ & References \\
\hline \multicolumn{5}{|c|}{ Known ACM genes coding for desmosome proteins } \\
\hline$P K P 2$ & Plakophilin-2 & $12 \mathrm{p} 11.21$ & $30-40$ & [7] \\
\hline$D S P$ & Desmoplakin & $6 \mathrm{p} 24.3$ & $10-15$ & [6] \\
\hline$D S G 2$ & Desmoglein-2 & $18 \mathrm{q} 12.1$ & $3-8$ & {$[8]$} \\
\hline$D S C 2$ & Desmocollin-2 & $18 \mathrm{q} 12.1$ & $1-5$ & [9] \\
\hline$J U P$ & Plakoglobin & $17 \mathrm{q} 21.2$ & $<1$ & {$[5]$} \\
\hline \multicolumn{5}{|c|}{ Other potential genes in ACM } \\
\hline CTNNA3 & Alpha T-catenin & $10 \mathrm{q} 21.3$ & $<1$ & [12] \\
\hline $\mathrm{CDH} 2$ & N-Cadherin & $18 \mathrm{q} 12.1$ & $<1$ & {$[13 *]$} \\
\hline TMEM43 & Transmembrane protein 43 & $3 \mathrm{p} 25.1$ & $<1$ & [14] \\
\hline$T T N$ & Titin & $2 q 31.2$ & $<1$ & {$[15]$} \\
\hline$F L N C$ & Filamin C & $7 \mathrm{q} 32.1$ & $<1$ & {$\left[16^{* * *}\right]$} \\
\hline LMNA & Lamin $\mathrm{A} / \mathrm{C}$ & $1 \mathrm{q} 22$ & $<1$ & {$[17]$} \\
\hline$T G F-\beta 3$ & Transforming growth factor $\beta 3$ & $14 \mathrm{q} 24.3$ & $<1$ & [18] \\
\hline$P L N$ & Phospholamban & $6 q 22.31$ & $<1$ & [19] \\
\hline$D E S$ & Desmin & $2 q 35$ & $<1$ & [20] \\
\hline$S C N 5 A$ & $\begin{array}{l}\text { Voltage-gated sodium channel } \\
\text { subunit alpha Nav1.5 }\end{array}$ & $3 p 22.2$ & $<1$ & {$[21 *]$} \\
\hline
\end{tabular}

